\title{
Homogeneity of spacetime implies the free Schrödinger equation
}

\author{
Shan Gao*
}

December 27, 2013

\begin{abstract}
The free Schrödinger equation is shown to be a consequence of spacetime homogeneity in the non-relativistic domain. This may help understand the origin of the wave equations in quantum theory.
\end{abstract}

\section{Introduction}

It is a fundamental assumption in modern physics that space and time are both homogeneous. The homogeneity of space and time is reflected in the spacetime translation invariance of natural laws, and it ensures that the same experiment performed at two different places or repeated at two different times gives the same result. In this paper, we will demonstrate that the free Schrödinger equation in quantum mechanics is a consequence of spacetime homogeneity, and it may be derived in terms of spacetime translation invariance when assuming linearity of time evolution.

The plan of this paper is as follows. In Section 2, spacetime translation invariance is analyzed. It is well known that spacetime translation gives the definitions of momentum and energy in quantum mechanics, and the momentum operator $P$ and energy operator $H$ are defined as the generators of space translation and time translation, respectively. Here we show that spacetime translation invariance entails that the state of a free particle with definite momentum and energy assumes the plane wave form $e^{i(p x-E t)}$ when assuming the time evolution of the state is linear. In Section 3, we show that conservation of energy and momentum, which is a consequence of spacetime translation invariance, may further determine the energy-momentum relation. In the nonrelativistic domain, the relation is $E=p^{2} / 2 m$, where $m$ is a constant property of the particle. In Section 4, we obtain the free particle Schrödinger equation based on these results. Conclusions are given in the last section.

*Institute for the History of Natural Sciences, Chinese Academy of Sciences, Beijing 100190, P. R. China. E-mail: gaoshan@ihns.ac.cn 


\section{Spacetime translation invariance}

There are in general two different pictures of translation: active transformation and passive transformation. The active transformation corresponds to displacing the studied system, and the passive transformation corresponds to moving the coordinate system. Physically, the equivalence of the active and passive pictures is due to the fact that moving the system one way is equivalent to moving the coordinate system the other way by an equal amount. In the following, we will mainly analyze spacetime translations in terms of active transformations.

A space translation operator can be defined as

$$
T(a) \psi(x, t)=\psi(x-a, t) .
$$

It means translating rigidly the state of a system, $\psi(x, t)$, by an amount $a$ in the positive $x$ direction. We assume $\psi(x, t)$ is an analytic function with respect to both $x$ and $t$. Then $T(a)$ can be further expressed as

$$
T(a)=e^{-i a P},
$$

where $P$ is called the generator of space translation ${ }^{1}$ By expanding $\psi(x-a, t)$ in order of $a$, we can further get

$$
P=-i \frac{\partial}{\partial x}
$$

Similarly, a time translation operator can be defined as

$$
U(t) \psi(x, 0)=\psi(x, t) .
$$

Let the evolution equation of state be of the following form:

$$
i \frac{\partial \psi(x, t)}{\partial t}=H \psi(x, t) .
$$

where $H$ is a to-be-determined operator that depends on the properties of the system ${ }^{2}$. Then the time translation operator $U(t)$ can be expressed as $U(t)=$ $e^{-i t H}$, and $H$ is the generator of time translation. In the following analysis, we assume $H$ is a linear operator independent of the evolved state, namely the evolution is linear 3

Let's now see the implications of spacetime translation invariance ${ }^{4}$ First, time translational invariance requires that $H$ have no time dependence, namely

\footnotetext{
${ }^{1}$ For convenience of later discussions we introduce the imaginary unit $i$ in the expression This does not influence the validity of the following analysis.

${ }^{2}$ Similarly we also introduce the imaginary unit $i$ in the equation for convenience of later discussions.

${ }^{3}$ Note that the linearity of $H$ is an important presupposition in our derivation of the free Schrödinger equation. It can be reasonably assumed that the linear evolution and nonlinear evolution both exist, and moreover, they satisfy spacetime translation invariance respectively because they cannot counteract each other in general. Then our following analysis will show that the linear evolution part, if it exists, must assume the same form as the free Schrödinger equation in the nonrelativistic domain. Certainly, our derivation cannot exclude the existence of possible nonlinear evolution.

${ }^{4}$ The evolution law of an isolated system satisfies spacetime translation invariance due to the homogeneity of space and time. The homogeneity of space ensures that the same experiment performed at two different places gives the same result, and the homogeneity in time ensures that the same experiment repeated at two different times gives the same result.
} 
$d H / d t=0$. This can be demonstrated as follows (see also Shankar 1994). Suppose an isolated system is in state $\psi_{0}$ at time $t_{1}$ and evolves for an infinitesimal time $\delta t$. The state of the system at time $t_{1}+\delta t$, to first order in $\delta t$, will be

$$
\psi\left(x, t_{1}+\delta t\right)=\left[I-i \delta t H\left(t_{1}\right)\right] \psi_{0}
$$

If the evolution is repeated at time $t_{2}$, beginning with the same initial state, the state at $t_{2}+\delta t$ will be

$$
\psi\left(x, t_{2}+\delta t\right)=\left[I-i \delta t H\left(t_{2}\right)\right] \psi_{0}
$$

Time translational invariance requires the outcome state should be the same:

$$
\psi\left(x, t_{2}+\delta t\right)-\psi\left(x, t_{1}+\delta t\right)=i \delta t\left[H\left(t_{1}\right)-H\left(t_{2}\right)\right] \psi_{0}=0
$$

Since the initial state $\psi_{0}$ is arbitrary, it follows that $H\left(t_{1}\right)=H\left(t_{2}\right)$. Moreover, since $t_{1}$ and $t_{2}$ are also arbitrary, it follows that $H$ is time-independent, namely $d H / d t=0$. It can be seen that this result relies on the linearity of time evolution. If $H$ depends on the state, then obviously we cannot obtain $d H / d t=0$ because the state is time-dependent, though we still have $H\left(t_{1}, \psi_{0}\right)=H\left(t_{2}, \psi_{0}\right)$, which means that the state-dependent $H$ also satisfies time translational invariance.

Secondly, space translational invariance requires $[T(a), U(t)]=0$, which further leads to $[P, H]=0$. This can be demonstrated as follows (see also Shankar 1994). Suppose at $t=0$ two observers $A$ and $B$ prepare identical isolated systems at $x=0$ and $x=a$, respectively. Let $\psi(x, 0)$ be the state of the system prepared by $A$. Then $T(a) \psi(x, 0)$ is the state of the system prepared by B, which is obtained by translating (without distortion) the state $\psi(x, 0)$ by an amount $a$ to the right. The two systems look identical to the observers who prepared them. After time $t$, the states evolve into $U(t) \psi(x, 0)$ and $U(t) T(a) \psi(x, 0)$. Since the time evolution of each identical system at different places should appear the same to the local observers, the above two systems, which differed only by a spatial translation at $t=0$, should differ only by the same spatial translation at future times. Thus the state $U(t) T(a) \psi(x, 0)$ should be the translated version of $A$ 's system at time $t$, namely we have $U(t) T(a) \psi(x, 0)=T(a) U(t) \psi(x, 0)$. This relation holds true for any initial state $\psi(x, 0)$, and thus we have $[T(a), U(t)]=0$, which says that space translation operator and time translation operator are commutative. Again, we note that the linearity of time evolution is an important presupposition of this result. If $U(t)$ depends on the state, then the space translational invariance will only lead to $U(t, T \psi) T(a) \psi(x, 0)=T(a) U(t, \psi) \psi(x, 0)$, from which we cannot obtain $[T(a), U(t)]=0$.

When $d H / d t=0$, the solutions of the evolution equation Eq.(5) assume the basic form

$$
\psi(x, t)=\varphi_{E}(x) e^{-i E t}
$$

and their linear superpositions, where $E$ is an eigenvalue of $H$, and $\varphi_{E}(x)$ is an eigenfunction of $H$ and satisfies the time-independent equation:

$$
H \varphi_{E}(x)=E \varphi_{E}(x) .
$$

The commutative relation $[P, H]=0$ further implies that $P$ and $H$ have common eigenfunctions. Since the eigenfunction of $P \equiv-i \frac{\partial}{\partial x}$ is $e^{i p x}$ (except a 
normalization factor $5^{5}$, where $p$ is an eigenvalue, the basic solutions of the evolution equation Eq.55 for an isolated system assume the form $e^{i(p x-E t)}$, which represents the state of an isolated system with definite properties $p$ and $E$. In quantum mechanics, $P$ and $H$, the generators of space translation and time translation, are also called momentum operator and energy operator, respectively. Correspondingly, $e^{i(p x-E t)}$ is the eigenstate of both momentum and energy, and $p$ and $E$ are the corresponding momentum and energy eigenvalues, respectively. Then the state $e^{i(p x-E t)}$ describes an isolated system (e.g. a free electron) with definite momentum $p$ and energy $E$.

\section{Conservation of energy and momentum and the energy-momentum relation}

In this section, we will show that the conservation of energy and momentum for an elastic collision between two systems in momentum eigenstates, which is a consequence of spacetime translation invariance, may further determine the energy-momentum relation for an isolated system being in a momentum eigenstate.

As we have demonstrated above, time translational invariance requires that $H$ has no time dependence, namely $d H / d t=0$, and space translational invariance requires that the generators of space translation and time translation are commutative, namely $[P, H]=0$. Then by Ehrenfest's theorem for an arbitrary observable $A$ and a normalized state $\psi(x, t)$

$$
\frac{d\langle A\rangle}{d t}=\left\langle\frac{\partial A}{\partial t}\right\rangle-i\langle[A, H]\rangle,
$$

where $\langle A\rangle=\int \psi^{*}(x, t) A \psi(x, t) d x$ is defined as the expectation value of $A$, we have

$$
\frac{d\langle H\rangle}{d t}=0
$$

and

$$
\frac{d\langle P\rangle}{d t}=0 .
$$

This means that the expectation values of energy and momentum are conserved for the evolution of an isolated system. In particular, for an isolated system being in a momentum eigenstate, its total momentum is not changed with time.

Before deriving the energy-momentum relation, we need to analyze velocity and velocity transformation. The velocity of a particle in an eigenstate $e^{i(p x-E t)}$ or a wavepacket superposed by these eigenstates is defined as the group velocity of the wavepacket, namely

$$
v=\frac{d E}{d p},
$$

By this definition, the velocity of the particle is always directed in the direction of its momentum. Moreover, the vector velocity can be written as a function of the vector momentum for a momentum eigenstate $e^{i(p x-E t)}$ :

\footnotetext{
${ }^{5}$ Strictly speaking, $e^{i p x}$ is a generalized eigenfunction in a $L^{2}$-space.
} 


$$
v=p / m(|p|),
$$

where $m(|p|)$ is a property of the particle that may depend on the absolute value of the momentum of the particle. Correspondingly, by solving Eq. (14) the energy of the particle will be $E=E(m,|p|)$, and it depends on both $|p|$ and $m$.

Now consider an elastic collision between two particles, which are in momentum eigenstates and move toward each other with exactly equal momenta and energies. Their total momentum is zero, and their speeds are the same. After the collision, it is possible that the momenta of the two particles are reflected at a certain angle relative to the initial direction, but momentum conservation (for these two particles as an isolated system) requires that their directions must be exactly opposite to each other, and they must also have the same values. Moreover, we suppose the two particles have the same property $m$. Then energy conservation further requires that their momenta must have the same values as their initial momenta. When considering the above velocity-momentum relation, this means that the corresponding velocities are also the same. Then by considering the non-relativistic velocity transformation from one inertial frame to another and requiring momentum conservation in all inertial frames (see Feynman, Leighton and Sands 1963 for a more detailed analysis) ${ }^{6}$ we can obtain the following relation in non-relativistic situations:

$$
m\left(\left|p_{1}\right|\right)=m\left(\left|p_{2}\right|\right),
$$

where $p_{1}$ and $p_{2}$ are two arbitrary momenta, which further implies

$$
v=p / m,
$$

where $m$ is a constant property of a particle independent of its momentum, and it is usually called the mass of the particle. Then we get the non-relativistic energy-momentum relation:

$$
E=p^{2} / 2 m \text {. }
$$

To sum up, the conservation of momentum and energy in all inertial frames may determine the energy-momentum relation, which is $E=p^{2} / 2 m$ in the non-relativistic domain.

\section{The free Schrödinger equation}

Since the operators $P$ and $H$ have common eigenfunctions for an isolated system, the relation between their eigenvalues $E$ and $p$ or the energy-momentum relation in the non-relativistic domain implies the operator relation $H=P^{2} / 2 m$ for an isolated system. By inputting this operator relation to the evolution equation

\footnotetext{
${ }^{6} \mathrm{By}$ using relativistic velocity transformation, we can obtain the relativistic energymomentum relation. Then, as we will show in the next section, the Klein-Gordon equation in relativistic quantum mechanics can be derived in terms of spacetime translation invariance. Since the Lorentz and Galilean transformations can be deduced based on homogeneity of space and time, isotropy of space and the principle of relativity (see, e.g. Pal 2003), this result indicates that the free wave equations in quantum theory are actually determined by certain properties of spacetime.
} 
Eq.(5), we can obtain the free evolution equation, which assumes the same form as the free particle Schrödinger equation in (nonrelativistic) quantum mechanics:

$$
i \frac{\partial \psi(x, t)}{\partial t}=-\frac{1}{2 m} \frac{\partial^{2} \psi(x, t)}{\partial x^{2}}
$$

Here it needs to be justified that the only parameter $m$ in this equation assumes real values; otherwise the appearance of the imaginative unit $i$ in the equation will be an illusion and the equation will be distinct from the free particle Schrödinger equation. Since velocity assumes real values, this is equivalent to proving that $p$ or the eigenvalue of the generator of space translation $P$ assumes real values, namely that the generator of space translation $P$ is Hermitian. This is indeed the case. Since the space translation operator $T(a)$ preserves the norm of the state: $\int_{-\infty}^{\infty} \psi^{*}(x, t) \psi(x, t) d x=\int_{-\infty}^{\infty} \psi^{*}(x-a, t) \psi(x-a, t) d x, T(a)$ is unitary, satisfying $T^{\dagger}(a) T(a)=I$. Thus the generator of space translation $P$, which is defined by $T(a)=e^{-i a P}$, is Hermitian.

In addition, it is worth noting that, unlike the free particle Schrödinger equation, the reduced Planck constant $\hbar$ with dimension of action is missing in this equation. However, this is in fact not a problem. The reason is that the dimension of $\hbar$ can be absorbed in the dimension of $m$. For example, we can stipulate the dimensional relations as $p=1 / L, E=1 / T$ and $m=T / L^{2}$, where $L$ and $T$ represents the dimensions of space and time, respectively (see Duff, Okun and Veneziano 2002 for a more detailed analysis). Moreover, the value of $\hbar$ can be set to the unit of number 1 in principle. Thus the above equation is essentially the free particle Schrödinger equation in quantum mechanics.

By using the definition of classical potential and requiring an appropriate expectation value correspondence, $d\langle P\rangle / d t=-\langle\partial V / \partial x\rangle$, we can further obtain the Schrödinger equation under an external potential $V(x, t)]^{7}$

$$
i \frac{\partial \psi(x, t)}{\partial t}=-\frac{1}{2 m} \frac{\partial^{2} \psi(x, t)}{\partial x^{2}}+V(x, t) \psi(x, t) .
$$

The general form of a classical potential may be $V\left(x, \frac{\partial}{\partial x}, t\right)$, and its concrete form is determined by the non-relativistic approximation of the quantum interactions involved, which are described by the relativistic quantum field theory.

\section{Conclusions}

The free Schrödinger equation in quantum mechanics is usually derived in textbooks by analogy and correspondence with classical mechanics (see, e.g. Schiff 1968; Landau and Lifshitz 1977; Greiner 1994) 8 , It begins with the assumption that the state of a free microscopic particle has the form of a plane wave

\footnotetext{
7 In order to derive the complete Schrödinger equation in a fundamental and rigorous way, we need a fundamental theory of interactions such as quantum field theory. It will be interesting to see whether the forms of basic interactions are also restricted or even determined by certain properties of space and time.

${ }^{8}$ There are also some attempts to derive the Schrödinger equation from Newtonian mechanics, one typical example of which is Nelson's stochastic mechanics (Nelson 1966). However, it has been argued that Nelson's derivation is problematic, and in particular, stochastic mechanics is inconsistent with quantum mechanics (Grabert, Hänggi, and Talkner 1979; Wallstrom 1994). In fact, Nelson himself also showed that there is an empirical difference between the predictions of quantum mechanics and his stochastic mechanics when considering quantum entanglement and nonlocality (Nelson 2005).
} 
$e^{i(k x-\omega t)}$. When combining with the de Broglie relations for momentum and energy $p=\hbar k$ and $E=\hbar \omega$, this state becomes $e^{i(p x-E t) / \hbar}$. Then it uses the nonrelativistic energy-momentum relation $E=p^{2} / 2 m$ to obtain the free particle Schrödinger equation. There are at least two mysteries in such a heuristic derivation. First, even if the behavior of microscopic particles likes wave and thus a wave function is needed to describe them, it is unclear why the wave function must assume a complex form. Indeed, when Schrödinger originally invented his equation, he was also puzzled by the inevitable appearance of the imaginary unit " $i$ " in the equation. Next, one doesn't know why there are the de Broglie relations for momentum and energy and why the nonrelativistic energymomentum relation is $E=p^{2} / 2 m$. Usually one can only resort to experience and classical physics to answer these questions. This seems unsatisfactory because quantum mechanics is generally regarded as a more fundamental theory, of which classical mechanics is only an approximation.

According to the above analysis, the key to unveil these mysteries is to analyze the homogeneity of space and time and the resulting spacetime translation invariance of natural laws. Spacetime translation gives the definitions of momentum and energy in quantum mechanics. The momentum operator $P$ is defined as the generator of space translation, and it is Hermitian and its eigenvalues are real. Moreover, the momentum operator can be uniquely determined by its definition, which turns out to be $P=-i \frac{\partial}{\partial x}$, and its eigenstate is $e^{i p x}$, where $p$ is the real eigenvalue. Similarly, the energy operator $H$ is defined as the generator of time translation. But its form is determined by the concrete situation. Fortunately, for an isolated system (e.g. a free microscopic particle) the form of energy operator, which determines the evolution equation, can be fixed for linear evolution by the requirements of spacetime translation invariance and relativistic invariance. Concretely speaking, time translational invariance requires that $d H / d t=0$, and thus the solutions of the evolution equation $i \frac{\partial \psi(x, t)}{\partial t}=H \psi(x, t)$ assume the form $\psi(x, t)=\varphi_{E}(x) e^{-i E t}$. Moreover, space translational invariance requires $[P, H]=0$, and this further entails that the state of a free particle with definite momentum and energy assumes the plane wave form $e^{i(p x-E t)}$. Furthermore, the relation between $p$ and $E$ or the energy-momentum relation can be determined by considering velocity transformation and conservation of momentum and energy, and in the nonrelativistic domain it is $E=p^{2} / 2 m$. Then we can obtain the energy operator for a free particle, $H=P^{2} / 2 m$, and the free particle Schrödinger equation, Eq. 19. This analysis might answer why the imaginary unit " $i$ " appears in the wave equation and why there are the de Broglie relations and why the nonrelativistic energy-momentum relation is what it is.

In conclusion, we have shown that the free Schrödinger equation may be regarded as a consequence of spacetime translation invariance when assuming linearity of time evolution. Though the requirements of these invariances are already well known, a strict derivation of the free Schrödinger equation in terms of them seems still missing in the literatur£? The new integrated analysis might help understand the origin of the wave equation in quantum mechanics.

\footnotetext{
${ }^{9}$ Note that some authors have derived the free Schrödinger equation in terms of Galilean invariance and a few other assumptions (Lévy-Leblond 1967; Musielak and Fry 2009), and these derivations are different from that given here.
} 


\section{References}

[1] Duff, M. J., Okun, L. B., and Veneziano, G. (2002). Trialogue on the number of fundamental constants. Journal of High Energy Physics, 03, 023.

[2] Feynman, R. P., Leighton, R. B., and Sands, M. (1963). The Feynman Lectures on Physics, vol. I. Reading: Addison-Wesley, pp. 16/6-16/7.

[3] Grabert, H., Hänggi, P., and Talkner, P. (1979). Is quantum mechanics equivalent to a classical stochastic process? Phys. Rev. A 19, 2440-2445.

[4] Greiner, W. (1994). Quantum Mechanics: An Introduction. New York: Springer.

[5] Landau, L. and Lifshitz, E. (1977). Quantum Mechanics. Oxford: Pergamon Press.

[6] Lévy-Leblond, J. M. (1967). Nonrelativistic particles and wave equations. Commun. Math. Phys. 6, 286.

[7] Musielak, Z. E. and Fry J. L. (2009). Physical theories in Galilean spacetime and the origin of Schrdinger-like equations. Annals of Physics 324, 296-308.

[8] Nelson, E. (1966). Derivation of the Schrödinger equation from Newtonian mechanics. Phys. Rev. 150, 1079-1085.

[9] Nelson, E. (2005). The mystery of stochastic mechanics, manuscript 200511-22.

[10] Pal, P. B. (2003). Nothing but relativity. Eur. J. Phys. 24, 315-319.

[11] Schiff, L. (1968). Quantum Mechanics. New York: McGraw-Hill.

[12] Shankar, R. (1994). Principles of Quantum Mechanics, 2nd ed. New York: Plenum.

[13] Wallstrom, T. (1994). Inequivalence between the Schrdinger equation and the Madelung hydrodynamic equations. Phys. Rev. A 49, 1613-1617. 